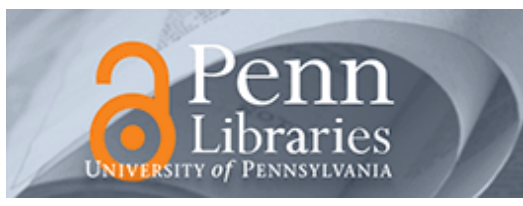

University of Pennsylvania ScholarlyCommons

$2-1985$

\title{
The Information Content of Specialist Pricing
}

John P. Gould

Robert E. Verrecchia

University of Pennsylvania

Follow this and additional works at: http://repository.upenn.edu/accounting_papers

Part of the Accounting Commons

\section{Recommended Citation}

Gould, J. P., \& Verrecchia, R. E. (1985). The Information Content of Specialist Pricing. Journal of Political Economy, 93 (1), 66-83.

Retrieved from http://repository.upenn.edu/accounting_papers/78

This article is available online at: http://www.jstor.org/stable/1830501

This paper is posted at ScholarlyCommons. http://repository.upenn.edu/accounting_papers/78

For more information, please contact repository@pobox.upenn.edu. 


\title{
The Information Content of Specialist Pricing
}

\begin{abstract}
This paper examines a process by which information-revealing prices are determined by considering the private incentives of a price-setting agent (whom we refer to as a specialist). The specialist has private information that may be (partially) revealed through his choice of a pricing rule. We define an equilibrium as a pricing rule and a response to that rule by a representative trader that maximizes the expected utilities of the specialist and the trader, conditional on each having rational expectations. By analyzing the existence and nature of this equilibrium, we attempt to develop further insights into the behavior of markets with incomplete information.
\end{abstract}

\section{Disciplines}

Accounting

\section{Comments}

This article is available online at: http://www.jstor.org/stable/1830501 


\section{The Information Content of Specialist Pricing}

\section{John P. Gould}

University of Chicago

\section{Robert E. Verrecchia}

University of Pennsylvania

This paper examines a process by which information-revealing prices are determined by considering the private incentives of a price-setting agent (whom we refer to as a specialist). The specialist has private information that may be (partially) revealed through his choice of a pricing rule. We define an equilibrium as a pricing rule and a response to that rule by a representative trader that maximizes the expected utilities of the specialist and the trader, conditional on each having rational expectations. By analyzing the existence and nature of this equilibrium, we attempt to develop further insights into the behavior of markets with incomplete information.

\section{Introduction}

The purpose of this paper is to deal simultaneously with two problems the literature on finance and economics has tended to address separately. On the one hand, the literature has considered the problem of determining the information content of prices without detailed modeling of the process by which prices are formed. On the other

An earlier draft of this paper was titled "The Specialist as Economic Agent." We greatly benefited from having had the opportunity to present earlier drafts of this paper at workshops sponsored by the University of British Columbia, UCLA, University of Chicago, Cornell, University of Houston, Purdue, Stanford, and University of Toronto. We would particularly like to acknowledge the remarks and advice of Douglas Diamond, Gerald Feltham, Mark Grinblatt, Sanford Grossman, Edward Lazear, Dale Morse, James Patell, José Scheinkman, Myron Scholes, and Mark Wolfson. 
hand, the literature has dealt with the process of price determination without paying much attention to the mechanism by which any information contained in prices satisfies a rational expectations equilibrium. ${ }^{1}$ By considering these two problems jointly, we hope to obtain further insights into how speculative markets work in a world of incomplete information.

Our approach is to analyze a model in which a specialist explicitly considers both the information content and the trading implications of the price he sets. Specifically, we consider a model of a market in which a specialist and a representative trader exchange a riskless numeraire good for a risky asset. Before trading occurs, both the specialist and trader are endowed with private information and assets. After trade occurs, the random return on the risky asset is realized, and the trader and specialist consume the liquidation value of the final portfolio they own. Trade itself is a two-step process. In step one, the specialist sets a price. ${ }^{2}$ In step two, the trader chooses the quantity he wishes to buy or sell at that price. ${ }^{3}$ Note that we restrict the analysis to a single-price case (e.g., multipart pricing or bid-ask spread pricing are not considered).

The specialist's selection of a price to maximize his expected surplus provides a rationale, or process, by which prices are formed. The specialist is not allowed to make the price contingent on the quantity purchased. Thus, in the second step in the trading process, the trader behaves as a pure price taker. In this way, we are able to link private incentives to set prices, through the introduction of the specialist, with the competitive behavior of traders exhibited in markets in which prices are set by a Walrasian auctioneer.

\footnotetext{
${ }^{1}$ No attempt is made here to provide a comprehensive listing of all the articles that have dealt with these topics. A partial listing of some of the relevant papers includes Kihlstrom and Mirman (1975), Garman (1976), Grossman (1976, 1978), Jaffe and Winkler (1976), Bradfield and Zabel (1979), Wilson (1979), Zabel (1979), Gould (1980), Grossman and Stiglitz (1980), Hellwig $(1980,1982)$, Verrecchia $(1980,1982)$, Diamond and Verrecchia (1981), Milgrom (1981), Grinblatt and Ross (1982), and Milgrom and Weber (1982).

${ }^{2}$ In practice, a specialist can play both an active role and a passive role in price determination. In his passive role, the specialist maintains a "book" of limit orders and fills market orders from this book at the price that is best from the viewpoint of the customer placing the market order. In his passive role, the specialist is purely a broker who is compensated by brokerage fees. In the active role, the specialist actually takes a market position himself. Our analysis considers only the active role of the specialist; we do not analyze the nature or behavior of limit orders. Moreover, we do not allow the specialist to limit his exposure-once he sets price he must balance the market by adjusting his own portfolio to cover any difference between the demand and supply quantities offered by traders. We make these abstractions to avoid unnecessary complications, not because we think that further extensions of the model would be uninteresting.

${ }^{3}$ We assume that the specialist can issue shares in either the risky or riskless asset, if necessary, to cover any excess or negative demand.
} 
If both the specialist and the trader had the same information, our problem would be similar to the standard textbook model of monopoly. ${ }^{4}$ This is not the case, however, when the specialist has private information, because then the trader's demand function depends on what he thinks the specialist uses to set prices. This means that some care needs to be taken in defining an equilibrium.

The price rule the specialist uses in setting a price potentially reveals his private information to the trader. The specialist selects a price to maximize his expected utility in anticipation of the trader's demand at that price, while carefully weighing the fact that the trader's demand is affected by what he learns from the price announcement. The trader, for his part, infers information on the basis of a conjecture about the price rule used by the specialist, which influences the trader's demand. Therefore, in our model, we define an equilibrium as a price rule and a response to that rule that maximizes the respective expected utilities of the specialist and trader conditional on each having rational expectations.

Because price reveals information, we assume that the specialist can disguise, or mask, whatever rate he selects for exchanging risky versus risk-free assets. The specialist does this by adding a noise term to the exchange rate he selects in determining the price he quotes the trader. ${ }^{5}$ Although we initially assume that the level of noise is an exogenously specified parameter, in Section IV we discuss what particular level of noise is optimal from the specialist's perspective.

A brief outline of the paper is as follows. In Section II we formally define an equilibrium to the problem outlined above. As a way of illustrating the nature of this equilibrium, in Section III we introduce specific assumptions that allow closed-form expressions. In Section IV, we examine the role of the level of noise in influencing the specialist's surplus and, in Section V, we consider the realism of our model in comparison with how a specialist is thought to operate. In the final section, we summarize our discussion.

\section{Definition of an Equilibrium}

In this section we define an equilibrium to a market for risky assets whose price is set by a maximizing monopolist. We assume that the

\footnotetext{
${ }^{4}$ The only difference, which is inconsequential, is that the quantity demanded may be negative-i.e., the trader may choose to sell rather than buy the risky asset.

${ }^{5}$ This raises an important, but subtle, modeling issue: if the trader acts as if the price quotation garbles the information the specialist has, then the specialist may either not garble the price or garble it in a way other than the trader is assuming. To avoid this inconsistency, we make one of two analytically equivalent assumptions: (1) there exists a precommitment mechanism that garbles the price, such as the static in the telephone line the specialist agrees to use in communicating the price to the trader, or (2) the parameters of the garbling distribution (the expression $V$ in our model) are revealed to the trader along with the noisy price.
} 
specialist is risk neutral: that is, he has a utility for consuming an amount $w$ represented by $S(w)=w$. The numeraire in the market is the price of a bond known to return one unit of the consumption good in the final period. The liquidating dividend on the risky asset is unknown until the final period and is represented by a random variable $\tilde{u}$ whose realization is denoted by $u$. (Henceforth, a tilde will be used to distinguish a random variable from its realization.) The trader is endowed with $B_{t}$ riskless assets and $x_{t}$ risky assets; the specialist's endowment is irrelevant (in this analysis) in the presence of his risk neutrality. The specialist and trader are also endowed with private information about the uncertain outcome $\tilde{u}$. Specifically, the specialist observes $\tilde{y}_{s}$ and the trader observes $\tilde{y}_{t}$, where each of $\tilde{y}_{s}$ and $\tilde{y}_{t}$ communicates the actual outcome $\tilde{u}=u$ perturbed by some noise.

We assume that in quoting a price to the trader, the specialist can precommit to garbling whatever value he selects as the appropriate rate of exchange for risky versus risk-free assets. Let $P\left(x_{t}, y_{s}\right)$ represent the value the specialist selects as the exchange rate as a function of the trader's endowment of the risky asset (which is common knowledge), $x_{t}$, and the private information observed by the specialist, $y_{s}$. Let $\tilde{\delta}$ represent the garbling, or noise, that is added to $P(\cdot)$. That is, the specialist selects $P\left(x_{t}, y_{s}\right)$, but the price quoted to the trader is $P\left(x_{t}, y_{s}\right)+$ $\tilde{\delta}$. Finally, let $R\left(x_{t}, B_{t}, y_{t}, p\right)$ represent the trader's excess demand for the risky asset, as a function of his endowment of the risky and riskfree assets, $x_{t}$ and $B_{t}$, respectively, his private information, $y_{t}$, and the price of the risky asset, $p$. This allows us to define expected utility functions $\gamma_{s}$ and $\gamma_{t}$, for the specialist and trader, respectively, by:

$$
\begin{gathered}
\gamma_{s}\left[x_{t}, y_{s}, p \mid R(\cdot)\right]=E\left[(p+\tilde{\delta}-\tilde{u}) R\left(x_{t}, B_{t}, y_{t}, p+\tilde{\delta}\right) \mid \tilde{y}_{s}=y_{s}\right], \\
\gamma_{t}\left[x_{t}, B_{t}, y_{t}, p, r \mid P(\cdot)+\tilde{\delta}\right]= \\
E\left\{U\left[(\tilde{u}-p)\left(r+x_{t}\right)+p x_{t}+B_{t}\right] \mid P\left(x_{t}, \tilde{y}_{s}\right)+\tilde{\delta}=p, \tilde{y}_{t}=y_{t}\right\},
\end{gathered}
$$

where $U(w)$ is the trader's utility for consuming an amount $w$. If, at $p$, the expression $R(\cdot)$ is positive, the specialist "covers" the trader by issuing shares against his own account of the risky asset sufficient to satisfy the excess demand; if the expression is negative, the specialist "eats" the excess supply by making it part of his personal portfolio.

This allows us to define an equilibrium to a market in which the price is set by a maximizing monopolist with private information as a pair of functions $P(\cdot)$ and $R(\cdot)$ such that

$$
\begin{gathered}
P\left(x_{t}, y_{s}\right)=\operatorname{argmax} \gamma_{s}\left[x_{t}, y_{s}, p \mid R(\cdot)\right], \\
R\left(x_{t}, B_{t}, y_{t}, p\right)=\operatorname{argmax} \gamma_{t}\left[x_{t}, B_{t}, y_{t}, p, r \mid P(\cdot)+\tilde{\delta}\right] .
\end{gathered}
$$

Essentially, $R(\cdot)$ is the set of arguments for $r$ that maximizes the trader's expected utility in response to any price $p$ he is quoted, given his 
conjecture about the price rule used by the specialist. $P(\cdot)$ is the set of arguments for $p$ that represents the specialist's best response to the trader's anticipated demand, given the private information the specialist observes. In this equilibrium, expectations are rational, or fulfilled, because the conditional expectation operators defining $\gamma_{s}$ and $\gamma_{t}$ are the correct ones based on the underlying joint distribution of $\tilde{u}, \tilde{y}_{s}, \tilde{y}_{t}$, and $\tilde{\delta}$.

\section{An Illustration of an Equilibrium}

To illustrate the nature of an equilibrium to the model we propose, we introduce three assumptions that facilitate the analysis. First, we assume that the random vector $\left(\tilde{u}, \tilde{y}_{s}, \tilde{y}_{t}, \tilde{\delta}\right)$ has a four-variate normal distribution with the mean $\left(y_{0}, y_{0}, y_{0}, 0\right)$ and covariance matrix

$$
\begin{gathered}
\tilde{u} \\
\tilde{y}_{s} \\
\tilde{y}_{t} \\
\tilde{\delta}
\end{gathered}\left[\begin{array}{lccc}
h^{-1} & h^{-1} & h^{-1} & 0 \\
h^{-1} & h^{-1}+f^{-1} & h^{-1} & 0 \\
0 & h^{-1} & h^{-1}+g^{-1} & 0 \\
& 0 & 0 & V
\end{array}\right]
$$

The expression $h$, which is the precision of the unconditional distribution of $\tilde{u}$, can be thought of informally as a measure of the amount of common knowledge about $\tilde{u}$. The expressions $f$ and $g$ can be interpreted as measures of the amount of private information held by the specialist and trader, respectively: for example, $f=0$ implies that the specialist has no information and $f \rightarrow \infty$ implies that he knows the realization $u$ of $\tilde{u}$ with certainty. The expression $V$ is the level of noise, or garbling, the specialist precommits to including in any price the trader is quoted.

Second, the trader has a (negative) exponential utility function with constant absolute risk tolerance of one: that is, he has a utility for consuming an amount $w$ represented by $U(w)=-\exp (-w)$. This, in conjunction with all the assumptions taken together and especially normality, implies that the trader's excess demand for the risky asset, $R(\cdot)$, is given by

$$
R\left(x_{t}, y_{t}, p\right)=\frac{E\left[\tilde{u} \mid \tilde{y}_{t}=y_{t}, P(\cdot)+\tilde{\delta}=p\right]-p}{\operatorname{var}\left[\tilde{u} \mid \tilde{y}_{t}=y_{t}, P(\cdot)+\tilde{\delta}=p\right]}-x_{t} .
$$

Note that for this case the trader's excess demand for the risky asset is independent of his endowment of the risk-free asset. ${ }^{6}$

\footnotetext{
${ }^{6}$ The discussion in this section is couched in terms of one trader facing the specialist. The results are easily generalized to many traders assuming that the traders' excess demand function is linear in price and all traders observe the same information. The model could be further generalized such that traders observe different information.
} 
Finally, we assume that the trader always conjectures that the rule used by the specialist in selecting price has the special linear form $P\left(x_{t}\right.$, $\left.y_{s}\right)=a y_{s}+b x_{t}+c$, where $a, b$, and $c$ are real-valued constants. Essentially, the effect of these three assumptions is to permit an explicit solution to the equilibrium concept we propose by preserving a certain linearity already inherent in the problem. ${ }^{7}$ In restricting attention to linear objective functions, we are not modifying our definition of equilibrium, but instead are looking for equilibria of a particular kind. We leave as unresolved the question whether nonlinear-type assumptions will also yield explicit solutions.

With regard to illustrating a solution to the special case we consider, our first result is to reduce the equilibrium concept to a more tractable form. Consider the specialist's choice of the parameters $a, b$, and $c$ in determining an expression for $P\left(x_{t}, y_{s}\right)$. Let $A(a)$ be a quadratic function of $a$ of the form $A(a)=a^{2}(h+f+g)-a f+V f(h+g)$.

Lemma 1: The existence of an equilibrium is equivalent to the existence of an $a^{*}$ that simultaneously satisfies

$$
[2 a(h+f)-f] A(a)-f g\left(V f+a^{2}\right)=0
$$

and

$$
A(a)>0 .
$$

Proof: See Appendix.

The intuition behind lemma 1 is that the existence of an equilibrium breaks down to two requirements: equation (1) ensures that the specialist's choice of $a^{*}$ leads to a conjecture that is fulfilled on the part of the trader, while equation (2) ensures that the specialist's choice of $a^{*}$ maximizes his expected utility (i.e., his objective function is globally concave with respect to $a$ ). The additional parameters $b^{*}$ and $c^{*}$ can be determined by substituting the value for $a^{*}$ into the functions $b(a)$ and $c(a)$ given by:

$$
b(a)=-\frac{a^{2}+f V}{2\left[a^{2}(h+f+g)+f V(h+g)\right]-f a} c(a)=y_{0}(1-a) .
$$

It is now possible to establish the existence of a unique equilibrium when $V>0, f>0$, and $g>0$.

Theorem 1: There exists a unique equilibrium.

Proof: First, observe that $a^{*}$ satisfies $[2 a(h+f)-f] A(a)-f g(V f+$ $\left.a^{2}\right)=0$ and $A(a)>0$ if and only if it is a root to the third-order

${ }^{7}$ Specifically, we have already assumed that the specialist is risk neutral and that garbling requires adding a noise term to the specialist's selection of price. 
polynomial $[2 a(h+f)-f] A(a)-f g\left(V f+a^{2}\right)$ in the region $[f / 2(h+$ $f), \infty)$. Then, observe that this third-order polynomial is

1. clearly negative at $a=f / 2(h+f)$;

2. eventually positive as $a$ becomes large, since the coefficient of $a^{3}$ is positive; and

3. convex in the region $[f / 2(h+f), \infty)$.

These three facts imply that the polynomial has a single (positive, real-valued) root in the region defined by $[f / 2(h+f), \infty)$. Therefore, there exists one, and only one, $a^{*}$ that both is a root to the polynomial expression and satisfies $A(a)>0$. Q.E.D.

Although the price the specialist quotes cannot be derived in a simple closed form, using lemma 1 straightforward expressions can be found for the three polar cases of $V=0, g=0$, or $f=0 .{ }^{8}$ The case $V=0$ is equivalent to assuming that the price the specialist selects is never masked.

Corollary 1: When $V=0$, a (unique) equilibrium takes the form:

$$
a^{*}=\frac{f}{h+f}, b^{*}=\frac{-1}{h+f+2 g}, c^{*}=\frac{h}{h+f} y_{0} .
$$

Proof: Suppose $V=0$. This reduces the requirement for an equilibrium in lemma 1 to finding an $a^{*}$ such that

$$
[2 a(h+f)-f] A(a)-f g a^{2}=0,
$$

and

$$
A(a)=a^{2}(h+f+g)-a f>0 .
$$

It is a simple exercise to show that a (unique) $a^{*}$ that satisfies (4) and (5) is given by $a^{*}=f /(h+f)$. This is because (4) reduces to a quadratic function with two real-valued roots, only one of which (i.e., $a=f /[h+$ f]) implies $A(a)>0$. Q.E.D.

Corollary 1 implies that in the absence of noise, that is, $V=0$, the specialist has no truly private information, since the trader can make unambiguous inferences about $\tilde{y}_{s}=y_{s}$ on the basis of $\tilde{P}=P$. Alternatively, a trader may have no private information whatsoever. This is equivalent to assuming $g=0$. When the specialist has private information while the trader does not, an equilibrium does not exist unless noise passes beyond some threshold.

Corollary 2: When $g=0$, a (unique) equilibrium exists only if $V>$ $f / 4 h(h+f)$; in that event, it takes the form

$$
a^{*}=\frac{f}{2(h+f)}, b^{*}=-\frac{1}{2}\left[\frac{1}{h}+\frac{f}{4 V h(h+f)^{2}}\right], c^{*}=\frac{2 h+f}{2(h+f)} y_{0} .
$$

${ }^{8} \mathrm{~A}$ closed-form solution can be found by solving the cubic equation implicit in the proof of theorem 1. However, this expression is not transparent. 
Proof: Suppose $g=0$. This reduces the requirement for an equilibrium in lemma 1 to finding an $a^{*}$ such that

$$
[2 a(h+f)+f] A(a)=0,
$$

and

$$
A(a)=a^{2}(h+f)-V f h-a f>0 .
$$

Here, a unique $a^{*}$ that satisfies $(6)$ is given by $a^{*}=f / 2(h+f)$. However, at that value, $A(a)$ is positive only if $V>f / 4 h(h+f)$. Thus, there exists no equilibrium when $0<V \leq f / 4 h(h+f)$. Q.E.D.

Corollary 2 has an immediate economic interpretation. Suppose that the trader conjectures that the price offered by the specialist contains information. If there is very little noise, that is, $V \leq f / 4 h(h+$ $f$ ), the trader puts much weight on what price he is quoted as a signal of the realization of the risky asset. For example, if a high price is quoted, the trader believes that the realization will be large and therefore demands a good deal of the risky asset. Thus, independent of his private information, the specialist's incentive is to select a high exchange rate, since if he can sell even a little bit of the risky asset at a very high price, the specialist makes a big profit. But if the specialist selects a high exchange rate independent of what he knows, price cannot contain information, so the trader's conjecture is false. Suppose, on the other hand, that the trader conjectures that price contains no information (i.e., he ignores price as a source of information). Then the (expected-utility-maximizing) exchange rate the specialist selects will depend on his private information, so the trader's conjecture is false once again. In short, there is no conjecture the trader can make that is fulfilled until noise passes beyond some threshold, which lessens the weight he puts on price as a source of information.

Corollary 2 is interesting also because of the discontinuity it suggests. Provided that the trader has some private information, that is, $g$ $>0$, no matter how small, an equilibrium exists independent of $V$. (For example, as shown in corollary 1 , it exists even at $V=0$.) The intuition here is that whenever the trader has some independent source of information to substantiate claims by the specialist, implicit in the price the trader is quoted, the specialist is kept in check and the equilibrium is preserved.

For completeness, we consider the case in which the specialist has no private information whatever, that is, $f=0$. This is equivalent to requiring that $a^{*}=0$ in the expression for price, since price cannot depend on information the specialist does not have.

Corollary 3: When $f=0$, a (unique) equilibrium takes the form $a^{*}=0, b^{*}=-[1 / 2(h+g)], c^{*}=y_{0}$. 
Proof: Since the specialist has no private information, $a^{*}$ must be zero. When $a^{*}$ and $f$ are zero, equations (1) and (2) are both implicitly satisfied (see the proof of lemma 1 in the App.). Substituting $a^{*}$ equal to zero into the expressions for $b$ and $c$ in (3) yields the result (factoring out $f$ in the former case). Q.E.D.

Corollary 3 is a straightforward monopoly problem since the specialist is endowed with no private information. For example, here the trader ignores price as a source of information, and thus his demand depends only on what he observes privately, $\tilde{y}_{t}=y_{t}$.

\section{The Specialist's Surplus}

The specialist's surplus is defined as the difference between the specialist's expected utility at an equilibrium and his expected utility at his autarky position. ${ }^{9}$ Because the specialist is risk neutral, we assume without loss of generality that the specialist's expected utility at his autarky position is zero. The purpose of this section is to explore how a specialist's surplus is affected by (1) the amount of the specialist's private information, as represented by $f ;(2)$ the amount of the trader's private information, as represented by $g$; and (3) the level of noise, as represented by $V$. The reason for examining this issue is that a specialist may be able to control or influence these parameters (especially $f$ and $V$ ); therefore, it is worth considering his incentives to do so. For example, a specialist may be able to control the amount of private information he acquires; or, if $V$ is thought of informally as the level of static in the telephone line over which he communicates the price of a risky asset, the specialist may be able to set the level of static to suit his purposes. To address this question, we first need an expression for the specialist's surplus.

Theorem 2: The specialist's surplus is determined by substituting the $a^{*}$ that satisfies equations (1) and (2) in lemma 1 into the expression

$$
\begin{gathered}
x_{t}^{2}\left\{\frac{\left(a^{2}+f V\right)\left[a^{2}(h+f+g)+f V(h+g)-f a\right]}{\left[2 a^{2}(h+f+g)+2 f V(h+g)-f a\right]^{2}}\right\} \\
+\frac{\left[a^{2}\left(h^{-1}+f^{-1}\right)-V\right]\left[a^{2}(h+f+g)+f V(h+g)-f a\right]}{a^{2}+f V}-g h^{-1} .
\end{gathered}
$$

Proof: See Appendix.

The usefulness of theorem 2 is limited because for the general case of $f, g$, and $V$, each positive, the parameter $a^{*}$ cannot be expressed in a

\footnotetext{
${ }^{9}$ Other definitions of the specialist's surplus might be used depending on what one assumes about the specialist's alternatives. In this definition, we implicitly assume that the specialist's alternative is not to trade at all (autarky).
} 
simple closed form. As a way of approaching the general case, we first consider, separately, the two polar cases in which (1) the specialist has no private information while the trader does (i.e., $f=0, g>0$ ); and (2) the trader has no private information while the specialist does (i.e., $f>0, g=0)$. Here, simple closed-form expressions for $a^{*}$ can be derived (see corollaries 2 and 3 above). For each of these cases, a recurrent question is what level of noise $V$ maximizes the specialist's surplus.

The case where the specialist has no private information and the trader does (i.e., $f=0, g>0$ ) helps to illuminate certain aspects of the model. One might suspect that the specialist would be at such an enormous disadvantage relative to the trader in this case that he would stay at his autarky position rather than trade at all. When $f=0$, the specialist's surplus is $\left[x_{t}^{2} / 4(h+g)\right]-(h+g) V-g h^{-1}$. It follows immediately from this equation that any positive value of $V$ makes the specialist worse off than when $V=0$. This result is actually quite plausible; noise interferes only with the specialist's ability to set the optimal price and cannot dilute the trader's information, because when $f=0$ the trader ignores price as a source of information anyway. When $V=0$, the specialist's surplus is $\left[x_{t}^{2} / 4(h+g)\right]-g h^{-1}$. This surplus is clearly decreasing in $g$, so the specialist is indeed worse off the better the quality of information available to the trader. However, if $g$ is small enough, in particular when $g<\left(-h+\sqrt{h^{2}+h x_{t}^{2}}\right) / 2$, the specialist's surplus will be positive and he will prefer to trade despite his severe information disadvantage.

This case illustrates the importance in this analysis of our assumption that the specialist is more risk tolerant than the trader. The trader's greater aversion to risk relative to the specialist means that the trader is willing to trade to get rid of some of his risky endowment even though the specialist sets the price of the risky asset below its expected value. This is analogous to the standard monopoly problem in which the seller gains by selling insurance at a monopoly price but loses because of inside information.

When the trader has no information and the specialist does, that is, $f>0, g=0$, theorem 2 implies that the specialist's surplus reduces to

$$
\frac{x_{t}^{2}}{4 h}-\frac{x_{t}^{2} f^{2}[4 V(h+f)+1]}{64 V^{2} h^{2}(h+f)^{3}}-\frac{[f-4 V h(h+f)]^{2}}{4 h\left[f+4 V(h+f)^{2}\right]} .
$$

Casual inspection of equation (8) suggests that for any $f>0$, there exists some $V$ in the interior of $[f / 4 h(h+f), \infty)$ that maximizes the specialist's surplus (where we consider only that region over which an equilibrium exists, i.e., $V>f / 4 h[h+f]$; see corollary 2). On closer inspection, however, we observe that no pair of $f>0$ and $V>f / 4 h(h$ $+f$ ) dominates $f=0$ and $V=0$ ! We state this as a corollary. 
Corollary 4: Provided that the trader has no information, the specialist has no incentive either to become informed or to introduce noise, when his alternative is a level of information and noise in a region over which an equilibrium exists.

Proof: Under the restriction $V>f / 4 h(h+f)$, the expression in equation (8) is (strictly) decreasing in $f$. Therefore, substituting the value $V=f / 4 h(h+f)$ into equation (8) achieves an upper bound on the specialist's surplus: namely, zero. However, when $f=0$ and $V=$ 0 , the expression in equation (8) assumes the value $x_{t}^{2} / 4 h$. Since this latter value is clearly higher, our claim is demonstrated. Q.E.D.

The conditioning statement in corollary 4 , namely, that the alternative to $f$ and $V$ equal to zero is some level at which an equilibrium exists, is based on technical rather than economic considerations. There is no endogenous motivation in the model for the specialist to guarantee an equilibrium when the trader has no information. ${ }^{10}$

Corollary 4 is useful for contrasting the polar case of $g=0$ with the more general case of $g>0$. The intuition underlying corollary 4 is that the specialist has no particular need for private information other than to inform him about the trader's excess demand function: if the trader has no private information, his excess demand function is known to the specialist with certainty. When the trader has some private information, it is generally the case that it is optimal for the specialist to be informed as well. The specialist profits from acquiring private information since this information tells him something about the excess demand to be submitted to him on the basis of the price he quotes.

It is also interesting to note from corollary 4 that $f=0, g=0$, and $V$ $=0$ is the symmetric information case, and here the specialist's surplus is $x_{t}^{2} / 4 h$. Clearly, the specialist's surplus decreases as $h$, the precision of the unknown liquidating value of the risky asset, increases. This suggests that as more information commonly known to the specialist and trader is made available, the rent earned by the specialist drops. This may help to explain the substantial unexpected drop of the price of a seat on the New York Stock Exchange in March 1934, the month when the Securities and Exchange Act of 1934 was introduced into Congress. If the Act had the effect of increasing information to traders, the seat price drop would be a reflection of the loss

\footnotetext{
${ }^{10}$ As discussed in Sec. III, when a trader has no information and attempts to use price as a source of information, the specialist's incentive is to choose an arbitrarily large price; however, it is not clear that this is economically meaningful in the absence of some constraint that ensures an equilibrium. If a regulatory authority prohibits the specialist from having private information (e.g., no "inside information"), then an equilibrium will exist and the specialist will choose $V=0$, even if the trader has no information. In this context, the regulatory prohibition on the specialist's having information might be thought of as a means of eliminating speculative bubbles.
} 
of specialist surplus. This point is more difficult to make in the more general expression that arises for the specialist's surplus in the diverse case (see theorem 2). Visual evidence of the seat price drop in March 1934 can be found in Schwert (1977).

With regard to an optimal level of noise $V$, we have shown so far that a specialist's surplus is maximized at $V=0$ when either $f=0$ or $g$ $=0$. Therefore, in these cases, garbling does the specialist no good. For each fixed and positive pair of $f$ and $g$, there generally exists a positive, finite level of noise $V$ that maximizes the specialist's surplus. ${ }^{11}$ On the one hand, the specialist profits from noise since this masks his private information from the trader. On the other hand, beyond a certain threshold, too much noise interferes with the specialist's ability to optimize.

\section{Consideration of Assumptions}

In summarizing our analysis, we consider the realism of our model in relation to how a specialist is commonly thought to operate. The first question concerns whether specialists are monopolists. Clearly, there is no prohibition on one trader's exchanging shares of a risky asset with some other trader and thereby circumventing the specialist. However, in the presence of large transaction costs associated with one trader's searching out someone else with whom to exchange assets at a mutually agreeable price, the idea that the trader will deal exclusively with the specialist is not unreasonable. Although these search costs are only implied in our model, when they are explicitly considered, the specialist may have the latitude to act as a monopolist.

The second question concerns the fact that price is not contingent on the quantity of shares exchanged. This has two implications: the specialist is put at some disadvantage in that he cannot use price as a barrier against a trader's private information, and the trader himself behaves as a pure price taker in that he believes his demand has no influence on price. With regard to the first implication, it is more reasonable to imagine that the specialist does make price contingent on the quantity traded. This would be a very simple mechanism to

\footnotetext{
${ }^{11}$ To show that there are situations in which the specialist prefers a positive, finite level of $V$, consider the case in which $f=g=h=1$. Here, we evaluate the specialist's surplus at $V=0, V=2$, and as $V$ approaches infinity. When $V=0$, the specialist's surplus is $0.0625 x^{2}-0.5$. When $V=2$, an equilibrium exists with $a=0.3823$ and the specialist's surplus can be written as $0.1206 x_{t}^{2}-4.2276$. As $V$ approaches infinity, $a$ approaches 0.375 , and the specialist's surplus approaches $0.125 x_{t}^{2}-2 \dot{V}-1$. It is a simple exercise to show that when $x_{t}>8.01$, the selection of $V=2$ dominates either $V$ zero or $V$ infinite. Since there exists some finite, positive level of $V$ (i.e., $V=2$ ) that yields a higher surplus than either polar extreme, the optimal level of $V$ (assuming the specialists can freely select it) must be finite and positive.
} 
guard against traders with superior information (e.g., "inside information") exploiting their knowledge. To that extent, our model suggests a limiting case to the more general circumstance in which prices are influenced by demand.

The second implication is, perhaps, more profound in that if traders are aware that their demand affects price, then they will behave strategically and not competitively. This fundamentally changes our analysis in that our primary objective is to integrate the role of the specialist without departing significantly from the competitive behavior exhibited by traders in the presence of a Walrasian auctioneer. We also remark that the large information requirements necessary for traders to act strategically suggest that competitive behavior is a more realistic approximation of an actual market setting.

The final question concerns whether specialists stay in business because they have a comparative advantage at bearing risk. (Looked at somewhat differently, the question could be rephrased to ask whether it would evolve naturally that the specialist would be the most risktolerant individual within a community of traders.) In our model, the specialist's greater tolerance for risk vis-à-vis the trader is key because it permits the specialist to achieve a positive surplus even in the presence of unfavorable information asymmetries. It can be argued, however, that the positive surplus that results from increased risk tolerance may proxy for the variety of institutional frictions (which we ignore) through which a specialist profits: transaction charges, a bidask spread, a price contingent on the quantity traded, etc. In other words, the specialist may indeed have no comparative advantage at bearing risk. However, the peripheral ways in which he earns rents by performing a specialist's tasks may cause him to behave as if he is more risk tolerant or at least ensure him a positive surplus even in the presence of better-informed traders.

\section{Conclusion}

This paper is motivated by what we perceive to be a gap in the literature on the determination of prices in rational expectations equilibria. Much of the research on rational expectations assumes the existence of a neoclassical Walrasian auctioneer who clears the market but is exogenous to the model. The Walrasian auctioneer assumption, though useful and powerful in many situations, is in a fundamental sense inconsistent with the spirit of rational expectations models, especially when information is not symmetrically distributed among market participants.

Our work yields a number of results of theoretical and empirical interest, the main points of which are summarized here. 
i) Equilibria exist under a broad set of conditions but not under all conditions. In particular, when the trader has no information and the specialist does, there is no equilibrium unless the noise in the price system is sufficiently large. When noise is small, a trader who attempts to use price as a source of information puts so much weight on the information contained in the price that the specialist has incentive to raise the price without bound.

ii) Assuming the trader has no information and that the specialist is forced to choose the level of noise sufficiently large to ensure an equilibrium, the specialist has no incentive either to acquire information or to introduce noise. Because the specialist is risk neutral, the only reason he is ever interested in acquiring information is to obtain a better estimate of the trader's demand function before setting price. When the trader has no information his demand function is perfectly predictable, so the specialist gains nothing from acquiring information. The optimal price is nonstochastic when the trader has no information, and it is only to the specialist's disadvantage to add noise.

iii) Even when the specialist is at an extreme information disadvantage relative to the trader (i.e., when the specialist has no private information but the trader does), the specialist may still prefer trading to autarky. This is because the specialist may be able to exploit the risk aversion of the trader sufficiently to offset the trader's information advantage.

iv) If both the specialist and the trader possess private information, the specialist may gain by adding a finite amount of noise to his price quotation. In this situation, the noise added to the price garbles the information transferred to the trader while forcing the specialist to relinquish some control over the price at which trading takes place.

Despite the restrictiveness of certain of our assumptions, we believe that our analysis has the advantage that it approaches the problem of modeling price formation from the correct methodological perspective. Specifically, the specialist's private incentives, in conjunction with the rational expectations of traders, are considered in providing a rationale for the information content of prices.

\section{Appendix}

\section{Proof of Lemma 1}

The proof of lemma 1 is in three parts. First, we determine the trader's optimal response to the offering of a price $p$ for the risky asset by computing his excess demand as a function of his conjecture about the price rule used by the specialist. Second, in part ii, we determine an expression for the exchange rate of the risky asset that maximizes the specialist's expected utility in anticipation of the trader's optimal response, and the information the specialist observes. Finally, in part iii, we show how the requirement that the trader's 
conjecture about the price rule be correct leads to the two expressions in lemma 1.

i) Suppose that the trader conjectures that the price rule has the following linear form: $\tilde{P}+\tilde{\delta}=a \tilde{y}_{s}+b x_{t}+c+\tilde{\delta}$. This implies that the random variables $(\tilde{u}, \tilde{y}, \tilde{P}+\tilde{\delta})$ have a trivariate normal distribution with mean $\left(y_{0}, y_{0}\right.$, $\left.a y_{0}+b x_{t}+c\right)$ and covariance matrix:

$$
\begin{aligned}
& \tilde{u} \\
& \tilde{y}_{t} \\
& \tilde{P}+\tilde{\delta}
\end{aligned}\left(\begin{array}{ccc}
h^{-1} & h^{-1} & a h^{-1} \\
h^{-1} & h^{-1}+g^{-1} & a h^{-1} \\
a h^{-1} & a h^{-1} & a^{2}\left(h^{-1}+f^{-1}\right)+V
\end{array}\right) .
$$

Then the trader's optimal response to the quotation of a price $p$ for the risky asset is an excess demand function of the form:

$$
R\left(x_{t}, y_{t}, p\right)=\frac{E\left[\tilde{u} \mid \tilde{y}_{t}=y_{t}, \tilde{P}(\cdot)+\tilde{\delta}=p\right]-p}{\operatorname{var}\left[\tilde{u} \mid \tilde{y}_{t}=y_{t}, \tilde{P}(\cdot)+\tilde{\delta}=p\right]}-x_{t},
$$

where $E\left[\tilde{u} \mid \tilde{y}_{t}=y_{t}, \tilde{P}(\cdot)+\tilde{\delta}=p\right]$ and $\operatorname{var}\left[\tilde{u} \mid \tilde{y}_{t}=y_{t}, \tilde{P}(\cdot)+\tilde{\delta}=p\right]$ are the mean and variance of $\tilde{u}$ conditional on observing $\tilde{y}_{t}=y_{t}$ and $P(\cdot)+\tilde{\delta}=p$. The mean and variance can be expressed as

$$
E\left[\tilde{u} \mid \tilde{y}_{t}=y_{t}, P(\cdot)+\tilde{\delta}=p\right]=y_{0}+g k_{1}\left(y_{t}-y_{0}\right)+k_{2}\left(p-a y_{0}-b x_{t}-c\right)
$$

and

$$
\operatorname{var}\left[\tilde{u} \mid \tilde{y}_{t}=y_{t}, P(\cdot)+\tilde{\delta}=p\right]=k_{1},
$$

where

$$
\begin{aligned}
& k_{1}=\frac{a^{2}+f V}{a^{2}(h+f+g)+f V(h+g)}, \\
& k_{2}=\frac{f a}{a^{2}(h+f+g)+f V(h+g)} .
\end{aligned}
$$

ii) The specialist observes the realization $\tilde{y}_{s}=y_{s}$ and then selects an exchange rate for the risky asset that maximizes his expected utility in anticipation of the trader's optimal response. This is equivalent to choosing a $p^{*}$ that solves

$$
\max _{p} E_{\{}(\tilde{u}-p-\tilde{\delta})\left[-R\left(x_{t}, \tilde{y}_{t}, p+\tilde{\delta}\right)\right]\left|\tilde{y}_{s}=y_{s}\right| \cdot
$$

The use of the calculus indicates that the first-order condition for a maximum is satisfied at $p^{*}$, where

$$
p^{*}=E\left(\tilde{u} \mid \tilde{y}_{s}=y_{s}\right)+\frac{E\left[R\left(x_{t}, \tilde{y}_{t}, p^{*}+\tilde{\delta}\right) \mid \tilde{y}_{s}=y_{s}\right]}{k_{1}^{-1}\left(1-k_{2}\right)},
$$

provided that $k_{2}<1$, thereby ensuring that the maximization problem is concave in $p$.

iii) The requirement $k_{2}<1$ becomes a requirement for an equilibrium, since in its absence, that is, $k_{2} \geq 1$, the specialist maximizes his expected utility by selecting $p^{*}$ infinite, independent of his private information. But if $p^{*}$ is always infinite, it does not depend on $\tilde{y}_{s}=y_{s}$ since it is invariant to the informa- 
tion observed by the specialist. Therefore, any conjecture the trader makes about price's being a linear function of the specialist's private information cannot be fulfilled in the absence of $k_{2}<1$. Note further that

$$
\begin{gathered}
E\left(\tilde{u} \mid \bar{y}_{s}=y_{s}\right)=y_{0}+\frac{f}{h+f}\left(y_{s}-y_{0}\right), \\
E\left[R\left(x_{t}, \tilde{y}_{t}, p+\tilde{\delta}\right) \mid \tilde{y}_{s}=y_{s}\right] \\
=k_{1}^{-1}\left[y_{0}+g k_{1} E\left(\tilde{y}_{t}-y_{0} \mid \tilde{y}_{\mathrm{s}}\right)+k_{2}\left(p-a y_{0}-b x_{t}-c\right)-p\right]-x_{t} \\
=k_{1}^{-1}\left\{y_{0}+g k_{1}\left[\frac{f}{h+f}\left(y_{s}-y_{0}\right)\right]-k_{2}\left(a y_{0}+b x_{t}+c\right)-\left(1-k_{2}\right) p\right\}-x_{t} .
\end{gathered}
$$

This implies that $p^{*}$ reduces to

$$
\begin{aligned}
p^{*}= & \frac{1}{2}\left(1+\frac{1}{1-k_{2}}\right) y_{0}+\frac{1}{2}\left(\frac{f}{h+f}\right)\left(1+\frac{g k_{1}}{1-k_{2}}\right)\left(y_{s}-y_{0}\right) \\
& -\frac{k_{1} x_{t}}{2\left(1-k_{2}\right)}-\frac{k_{2}\left(a y_{0}+b x_{t}+c\right)}{2\left(1-k_{2}\right)} .
\end{aligned}
$$

However, for the trader's conjecture to be fulfilled, it must be that $E\left(p^{*}+\tilde{\delta}\right)$ $=E\left(a \tilde{y}_{1}+b x_{t}+c+\tilde{\delta}\right)=a y_{0}+b x_{t}+c$. This requirement reduces $p^{*}$ further to

$$
p^{*}=y_{0}+\frac{1}{2}\left(\frac{f}{h+f}\right)\left(1+\frac{g k_{1}}{1-k_{2}}\right)\left(y_{s}-y_{0}\right)-\frac{k_{1} x_{t}}{2-k_{2}} .
$$

Therefore, an equilibrium is a triplet of constants:

$$
a=\frac{1}{2}\left(\frac{f}{h+f}\right)\left(1+\frac{g k_{1}}{1-k_{2}}\right), b=-\frac{k_{1}}{2-k_{2}}, c=(1-a) y_{0} .
$$

However, $k_{1}$ and $k_{2}$ are functions of $a$. Substituting in the expressions for $k_{1}$ and $k_{2}$, a solution to equation (Al) requires that $a$ be a real-valued root to the third-order polynomial equation

$$
[2 a(h+f)-f]\left[a^{2}(h+f+g)-a f+V f(h+g)\right]-f g\left(V f+a^{2}\right)=0 .
$$

Furthermore, the requirement $k_{2}<1$ is equivalent to

$$
a^{2}(h+f+g)-a f+V f(h+g)>0 .
$$

Q.E.D.

\section{Proof to Theorem 2}

Theorem 2 is simply a computation of the difference between the specialist's expected utility at an equilibrium and the specialist's autarky point. First, note that $\tilde{P}$ can be expressed as $\tilde{P}=a\left(\tilde{y}_{s}-y_{0}\right)+y_{0}+b x_{t}+\tilde{\delta}$, and $R(\cdot)$ as $R\left(x_{t}, \tilde{y}_{t}, p\right.$ 
$+\tilde{\delta})=k_{1}^{-1}\left\{g k_{1}\left(\tilde{y}_{t}-y_{0}\right)-b x_{t}-\left(1-k_{2}\right)\left[a\left(y_{s}-y_{0}\right)+\tilde{\delta}\right]\right\}-x_{t}$. Thus, the specialist's surplus is

$$
\begin{aligned}
& E_{\bar{y}_{i}}\left[E_{\bar{u}, \tilde{\delta}}\left(\left[\tilde{u}-a\left(y_{s}-y_{0}\right)-y_{0}-b x_{t}-\tilde{\delta}\right]\right\}-R\left[x_{t}, \tilde{y}_{t}, a\left(y_{s}-y_{0}\right)\right.\right. \\
& \left.\left.+y_{0}+b x_{t}+\tilde{\delta}\right]\left[\mid \tilde{y}_{s}=y_{s}\right)\right] \text {. } \\
& =-b x_{t}^{2}-k_{1}^{-1} E_{y_{s}}\left[E _ { \tilde { u } , \tilde { \hat { \delta } } } \left[\left(\left[\tilde{u}-y_{0}\right)-a\left(y_{s}-y_{0}\right)-b x_{t}-\tilde{\delta}\right]\right.\right. \\
& \text { - } \left.\left.\left\{g k_{1}\left(\tilde{y}_{t}-y_{0}\right)-b x_{t}-\left(1-k_{2}\right)\left[a\left(y_{s}-y_{0}\right)+\tilde{\delta}\right]\right\} \mid \tilde{y}_{s}=y_{s}\right)\right] \\
& =-x_{t}^{2} b\left(1+k_{1}^{-1} b\right)+a g h^{-1}+a\left(1-k_{2}\right) k_{1}^{-1} h^{-1}-a^{2}\left(1-k_{2}\right) k_{1}^{-1}\left(h^{-1}+f^{-1}\right) \\
& -\left(1-k_{2}\right) k_{1}^{-1} V-g h^{-1} \\
& =-x_{t}^{2} b\left(1+k_{1}^{-1} b\right)+a^{2}\left(1-k_{2}\right) k_{1}^{-1}\left(h^{-1}+f^{-1}\right)-\left(1-k_{2}\right) k_{1}^{-1} V-g h^{-1},
\end{aligned}
$$

where the last equality follows from the relation for an equilibrium required in equation (A2). Substituting back in the expressions for $k_{1}$ and $k_{2}$ (see the proof to lemma 1) yields the results. Q.E.D.

\section{References}

Bradfield, James, and Zabel, Edward. "Price Adjustment in a Competitive Market and the Securities Exchange Specialist." In General Equilibrium, Growth, and Trade, edited by Jerry R. Green and José A. Scheinkman. New York: Academic Press, 1979.

Diamond, Douglas W., and Verrecchia, Robert E. "Information Aggregation in a Noisy Rational Expectations Economy." J. Financial Econ. 9 (September 1981): 221-35.

Garman, Mark B. "Market Microstructure.” J. Financial Econ. 3 (June 1976): $257-75$.

Gould, John P. "The Economics of Markets: A Simple Model of the Market Making Process." J. Bus. 53, no. 3, pt. 2 (July 1980): S167-S187.

Grossman, Sanford J. "On the Efficiency of Competitive Markets Where Traders Have Diverse Information." J. Finance 31 (May 1976): 573-85.

- "Further Results on the Informational Efficiency of Competitive Stock Markets." J. Econ. Theory 18 (June 1978): 81-101.

Grossman, Sanford J., and Stiglitz, Joseph E. "On the Impossibility of Informationally Efficient Markets." A.E.R. 70 (June 1980): 393-408.

Grinblatt, Mark S., and Ross, Stephen A. "Market Power in a Securities Market with Endogenous Information." Working Paper. Los Angeles: UCLA, 1982.

Hellwig, Martin F. "On the Aggregation of Information in Competitive Markets." J. Econ. Theory 22 (June 1980): 477-98.

_ . "Rational Expectations Equilibrium with Conditioning on Past Prices: A Mean-Variance Example." J. Econ. Theory 26 (April 1982): 279-312.

Jaffe, Jeffrey F., and Winkler, Robert L. "Optimal Speculation against an Efficient Market." J. Finance 31 '(March 1976): 49-61.

Kihlstrom, Richard E., and Mirman, Leonard J. "Information and Market Equilibrium." Bell J. Econ. 6 (Spring 1975): 357-76.

Milgrom, Paul R. "Rational Expectations, Information Acquisition, and Competitive Bidding." Econometrica 49 (July 1981): 921-43.

Milgrom, Paul R., and Weber, Robert J. "A Theory of Auctions and Competitive Bidding." Econometrica 50 (September 1982): 1089-1122. 
Schwert, G. William. "Stock Exchange Seats as Capital Assets." J. Financial Econ. 4 (January 1977): 51-78.

Verrecchia, Robert E. "Consensus Beliefs, Information Acquisition, and Market Information Efficiency." A.E.R. 70 (December 1980): 874-84.

- "Information Acquisition in a Noisy Rational Expectations Economy." Econometrica 50 (November 1982): 1415-30.

Wilson, Robert. "Auctions of Shares." Q.J.E. 93 (November 1979): 675-89.

Zabel, Edward. "Competitive Price Adjustment without Market Clearing." Working Paper. Rochester, N.Y.: Univ. Rochester, 1979 (rev. 1980). 